\title{
Trimetrexate Glucuronate
}

National Cancer Institute

\section{Source}

National Cancer Institute. Trimetrexate Glucuronate. NCI Thesaurus. Code C1265.

A lipid soluble methotrexate derivative with potential antineoplastic activity. T rimetrexate glucuronate inhibits the enzyme dihydrofolate reductase, thereby preventing the synthesis of purine nucleotides and thymidylate, with subsequent inhibition of DNA and RNA synthesis. Trimetrexate glucuronate also exhibits antiviral activity. ( $\mathrm{NCI04)}$ 